\title{
Three-Dimensional Aerodynamic Shape Optimization of Supersonic Delta Wings
}

Greg W. Burgreen and Oktay Baysal

Old Dominion University

Norfolk, VA

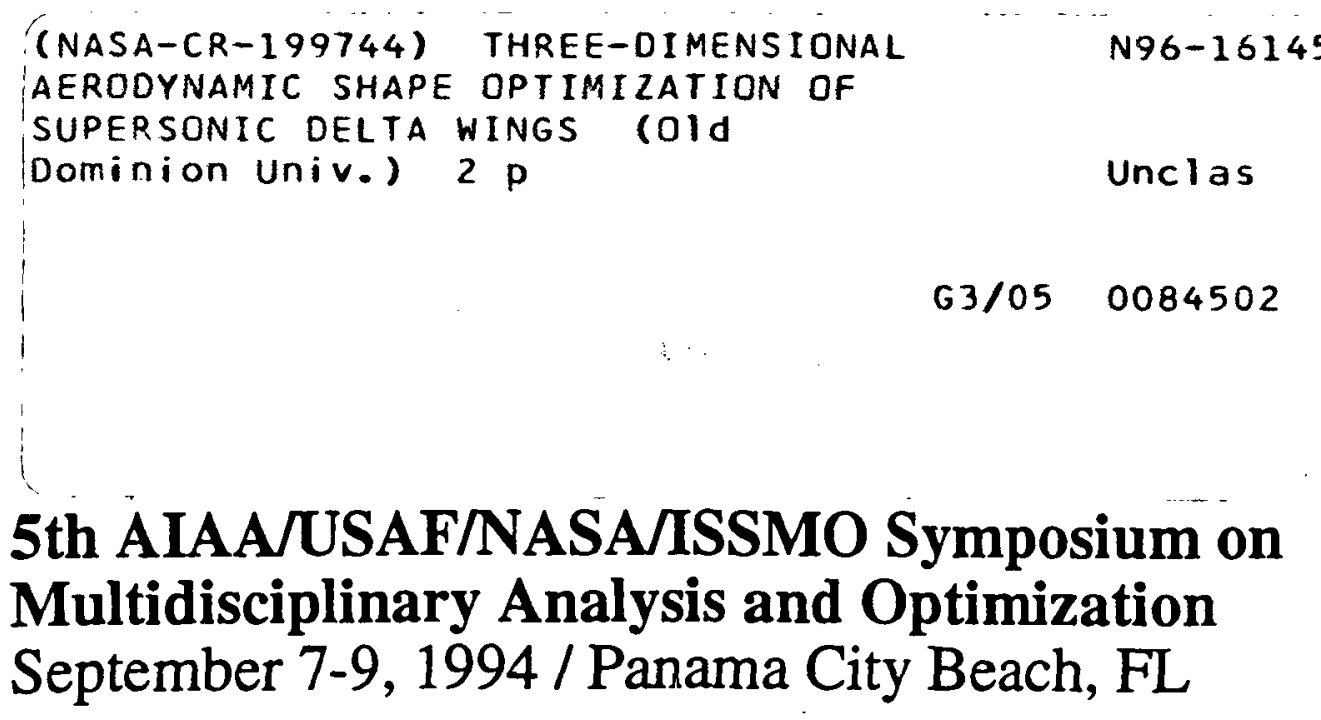

For permission to copy or republish, contact the American Institute of Aeronautics and Astronautics 370 L'Enfant Promenade, S.W., Washington, D.C. 20024 


\title{
Three-Dimensional Aerodynamic Shape Optimization of Supersonic Delta Wings
}

\author{
Greg W. Burgreen* and Oktay Baysal $\dagger$ \\ Old Dominion University, Norfolk, Virginia 23529
}

\begin{abstract}
A recently developed three-dimensional aerodynamic shape optimization procedure AeSOP $_{3 D}$ is described. This procedure incorporates some of the most promising concepts from the area of computational aerodynamic analysis and design, specifically, discrete sensitivity analysis, a fully implicit $3 D$ CFD methodology, and 3D Bezier-Bernstein surface parameterizations. The new procedure is demonstrated in the preliminary design of supersonic delta wings. Starting from a symmetric clipped delta wing geometry, a Mach 1.62 asymmetric delta wing and two Mach 1.5 cranked delta wings were designed subject to various aerodynamic and geometric constraints.
\end{abstract}

\section{Introduction}

The ingenuity and tenacity of aerodynamic designers have served them well over the history of aeronautics. Near-optimal designs for many aerodynamic components have been produced with the investment of many hours of analysis and experimental testing. An excellent example of this is the development of the supercritical airfoil shape by Whitcomb [1] in the 1960's. His superior insight and understanding of flow physics, in conjunction with extensive wind tunnel testing, guided the systematic evolution of this radically new and efficient airfoil shape.

Over the years, this heuristic type of approach has been successfully employed in the design of many complex configurations including complete aircraft whose behavior is a resultant of complex interactions among many different physical phenomena and hardware components. However, in the last decade, an emerging trend in the analytical design of complex engineering

\footnotetext{
*Graduate Research Assistant, School of Mechanical and Aerospace Engineering. Currently, Post-Doctoral Research Specialist, The University of Pittsburgh Medical Center. Student Member, AlAA.

$\dagger$ Professor, Dept. of Aerospace Engineering. Senior Member, AIAA.

Copyright (c) 1994 by G. W. Burgreen. Published by the American Institute of Aeronautics and Astronautics, Inc. with permission.
}

systems is the concurrent integration of all appropriate disciplines in the design process. This new discipline is referred to as Multidisciplinary Design Optimization (MDO) [2]. One of the principle components of $\mathrm{MDO}$ is sensitivity analysis, which quantifies the sensitivity of the system outputs to design changes. Intrinsic to the future success of MDO applications is the maturation of sensitivity analysis-based optimization procedures within the individual engineering disciplines. The present work concentrates on one such single disciplinary design topic-aerodynamic shape optimization.

One of the latest technological advances in the field of aerodynamic design optimization is the inclusion of discrete aerodynamic sensitivity analysis within the design process. In this context, the function of sensitivity analysis is to directly couple computational fluid dynamics (CFD) with numerical optimization techniques. This coupling facilitates the development of efficient directdesign procedures that have the capability to automatically determine optimal geometric surfaces that are not biased by intuition or experience in engineering.

Integration of the new discrete sensitivity analysis technology into a functional aerodynamic shape optimization procedure has only recently been accomplished by a few groups. These groups include for two-dimensional design: Drela [3], Baysal et al. [4-8], Taylor et al. [9], Grossman et al. [10], Ghattas et al. [11], and Huffman et al. [12]; and for three-dimensional design: Grossman et al. [13], and Baysal et al. $[14,15]$.

The results from these applications have indicated that, in general, the design methods obtain a final optimum aerodynamic shape via an evolution of successively improved shapes, although sometimes at a rather high computational cost due to the large number of flow analyses involved. In the present work, this high computational cost is reduced via the introduction of a truly practical use of fully implicit CFD methodologies (Newton's method) within the optimization procedure. 